\title{
Coal Mine Security System
}

\author{
Warsha M.Choudhari \\ Professor, Information Technology \\ Datta Meghe Institute of Engineering, Technology \& Research \\ Wardha, India
}

\begin{abstract}
Coal Mine Security System uses sensor, automatic detection, and communication and microcontroller 89S52 technologies, to realize the operational parameter intelligent monitored management of entire mining area. The data acquisition terminal take the 89S51 chip integrated circuit as a core, cooperating highly effective digital sensor, carries on the communication through the RS232 interface with the main control machine, which has realized the intelligent monitoring. Data management system. Use of EEPROM chip as a Black box to store data permanently, Database system used to stored data as backup. Also use CCTV camera for recording internal situation. use a new generation Matlab technique, which based on the Client/Server mode. The system implements the realtime monitoring and displaying for data under mine, deletion and maintenance of history data, graphic statistic, report printing, expert diagnosis and decision-making support modules. The intelligent decision-making support module sets in the system, can detect the level of parameters which are affected the production of coal mine as well as human being and the mechanical device breakdown troubleshooting, Also may in the accident situation, provides the intelligent decisionmaking to rescue and disperse the personnel and equipment. The Research, development and Promote Application will provide the safeguard regarding the mine pit security work. The paper shows that the system is flexible in the architecture of software and hardware, and can be easily extended to other mine safety production fields.
\end{abstract}

Keywords: Coal mine security system; Client/server mode; Intelligent decision-Making support; Matlab.

\section{INTRODUCTION}

Coal Enterprise is the high-risk profession and technique now is relative backwardness. Security is the most important in the coal mine production establishing mine safety production safeguard system is the only way to guarantee the safety in coal mine production. Currently in mine production, there are mainly following two aspects to impact the safety in mine production.

(1) Environment Parameter: Gas, Carbon Monoxide, Temperature (Humidity) Degree, Coal Position of the Bunker, Pressure of the roof etc.

(2) Electromechanical Device Running Parameters: transport fix, belt conveyer, Voltage, Electric Current and so on.

In summary, Coal mine security monitor system in coal mine is the significant measure that safeguards the safe production in coal mine. It acts vital role in disaster prevention and reduction in mine, as well as improves the productivity. It also is the significant milestone of implementing the modern management for mine production.

\section{SUMMARIZATION OF SYSTEM}

Coal Mine Security System mainly monitor the parameter in coal mine, like Gas( $\mathrm{CH} 4, \mathrm{CO}$, etc), Temperature, and so on as well as the main production equipment stop the switch parameter, forecast mine production security information, effectively avoid the occurrence of gas and coal dust explosion significant malignant accident. Compares with the former system, this system subordinate control computer has used the intelligent digital sensor, increased precision of the data acquisition, the expert system module, can provide the solution way when the mine exceptional operation.

1) Hardware part of the system is comprised of data acquisition terminal, data concentrator and main control computer.

2) Software part of the system is comprised of Mine Monitoring Data Management system based on Matlab, it is used for integrated management and monitoring of the whole mining area.

3) The whole system will transfer the real data to main control computer monitoring program through the RS232 communication interface, to display, store, query and print the mine environment parameters and kinds of electromechanical devices' running parameters.

\section{HARDWARE STRUCTURE OF SYSTEM}

The entire monitor system divides into the mine data acquisition terminal and the ground concentrator [3]. The realtime data in the mine pit transmit the dynamic analog signals to the subordinate substation cybertron through the intelligent numeral sensor, and backup to database, then through network data is transferred to main terminal data concentrator, implements the monitoring by the main terminal main engine. When has the accident, the main engine will enter reporting warning and ceases module automatically, carries on power failure processing to the electrical equipment, to reduce the loss in the greatest degree. Intelligent data gathering terminal program flow chart shown as in figure 1 . The procedure in the data gathering terminal uses the S52's language to compile mainly completes following several function:

(1) Each kind of sensor analog parameter gathering and A/D transformation, the data reads. 
(2) Gathering the value of open, stops, switch quantity and other electrical parameter to Control system.

(3) Data computation and memory, warning judgment, power source management a system self- check.

(4) Carries on the data exchange with the concentrator taking the power line as the medium through the carrier communication.

(5) To be possible to carry on the local data through the local serial port to copy reads and the parameter establishment.

The main control software part of the overall system installs on the main terminal computer as well as the subordinate substation various labor controls machine. The main interface is coded by the new generation Matlab. It can be divided into 6 parts for movement structure and 4 parts for databases. The real-time data acquisition display module has implemented the acquisition and display of all changed data of the entire mining area; The history data inquiry and the maintenance module can be used to manage and maintain all the collected data; The statistical history data graph module can be used to analyze and compare the historical data; The display and printing data report module is used to in print the mine data form; The expert decision-making module can complete the gas forecast, mechanical device breakdown diagnosis, accident decisionmaking; The warning and ceasing engine module can give the alarm timely when the data changed abnormally, disconnect the power source automatically to ensure the safety of equipment when the data surpass the predetermined value.

\section{OVERALL STRUCTURE OF SYSTEM}

\section{A. System Main Control Software Modular Structure}

The main control software part of the overall system installs on the main terminal computer as well as the subordinate substation various labor controls machine. The main interface is coded by using Matlab.

The real-time data acquisition display module has implemented the acquisition and display of all changed data of the entire mining area; The history data inquiry and the maintenance module can be used to manage and maintain all the collected data; The statistical history data graph module can be used to analyze and compare the historical data; The display and printing data report module is used to in print the mine data form; The expert decision-making module can complete the gas forecast, mechanical device breakdown diagnosis, accident decision-making; The warning and ceasing engine module can give the alarm timely when the data changed abnormally, disconnect the power source automatically to ensure the safety of equipment when the data surpass the predetermined value.

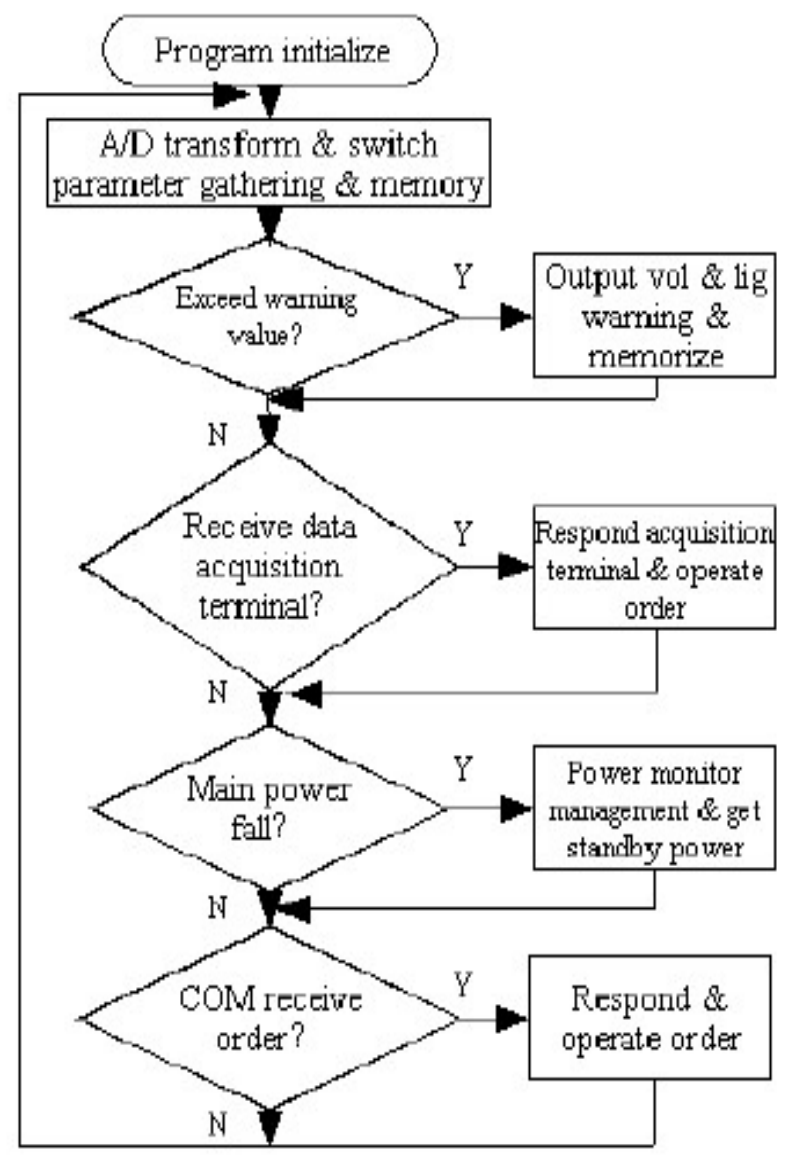

Fig 1 Intelligent Datat Gathering Terminal Progran Flow Chart

\section{B. Intelligent Decision-Making Module Design}

According to expert knowledge base, it can provide the suggestion and hints for variety parameter of abnormal parameter to forecast the shaft gas; can provide the best environment parameter and reasonable production instruction; indicate the best disaster relief and evades the disaster route and provide the decision-making for rescuing and dispersing personnel and equipment when disaster appearance. Shown as in figure 2.

\section{Human-Machine Interface}

Human-machine interface is the interface for watch to provide information to system, bring forward the task requirement and for system to provide solution and various assistant decision-making information to user.

\section{Intelligent Decision-Making Support System}

Its functions include data acquisition, data identification, data calculation, etc. In details, it will analyze and estimate the issues entered through human-machine interface, bring forward the relative solution, describe the issue formally, including the representation, organization, access and usage of data and knowledge. It also can use the knowledge provided by knowledge base to solve the issue, get the solution, and institute the decision-making mode for issue resolution, 
correspond the relationship of each subsystem and transfer the information.

\section{Knowledge Base Structure}

A like object-oriented knowledge representation, expert's knowledge expresses as class, property object and so on. Each knowledge base contains four classes: class table, property table, object table and multi-media information table, its structure is: class table (table (class name, parent class name, sub-class name, typical characteristics); property table (class name, property name, property type, value range); object table (class name, object name, property name, property value); multi-media information table (object name, picture, video).

\section{Client/Server mode}

Based on the Client/Server mode, coal mine safety monitor system connects each tache of coal mine closely through the network, enables the coal mine superintendent to control entire enterprise's production management in real-time. This mode applies Windows Socket which is core technology of network programming. The main station group network connected together through the net cable. As server, and the subordinate station network are the work master station has implemented seamless integration for the data and service with the subordinate substation client. It has openness and easy to expand and so on the merits. $\mathrm{T}$ It divides into the forestage client and the backstage server, to saves mine information parameters and management information decision-making separately.

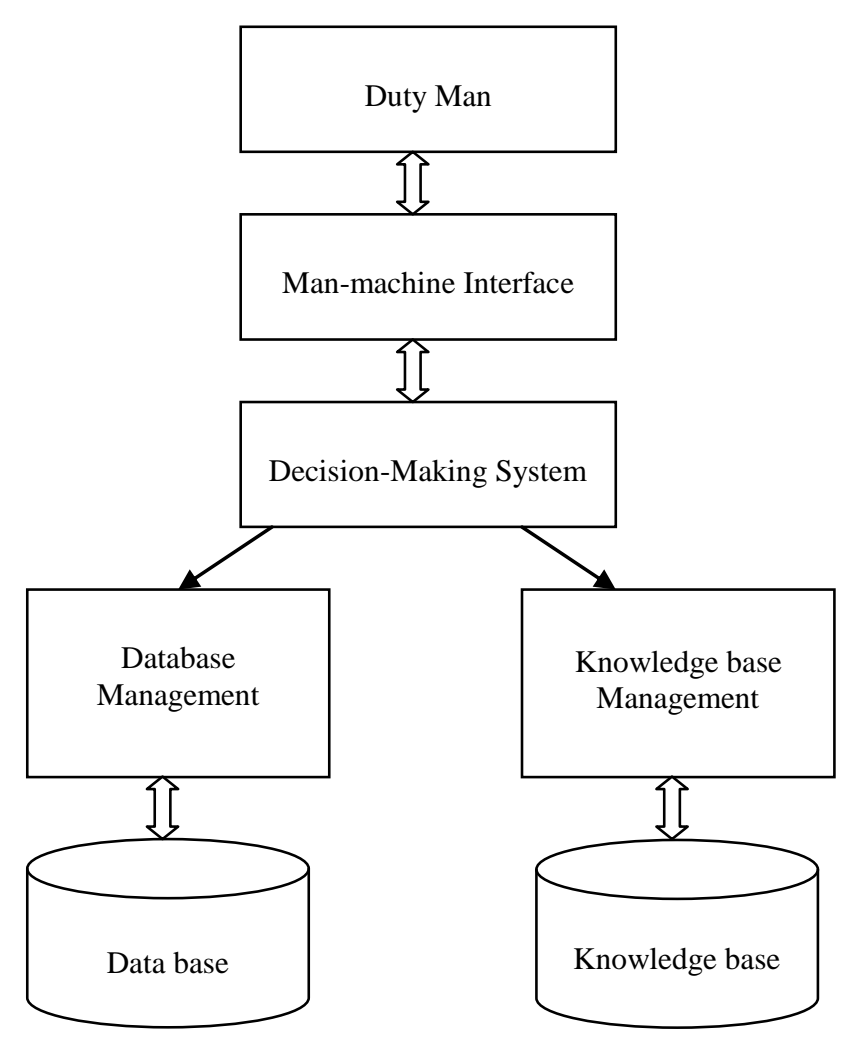

Fig 2 structure of expert decision-making and consultation system

\section{Prompt Data Collection Technology}

Data collection means that the data from the hardware system is converted into available data, while data can be written to the hardware system so as to establish the control and application the two-way connection software. Data management is analyzing and dealing with the data with the application procedure when receive data. The core of data collection and data management is prompt Poisoning accident disaster prevention and mitigation

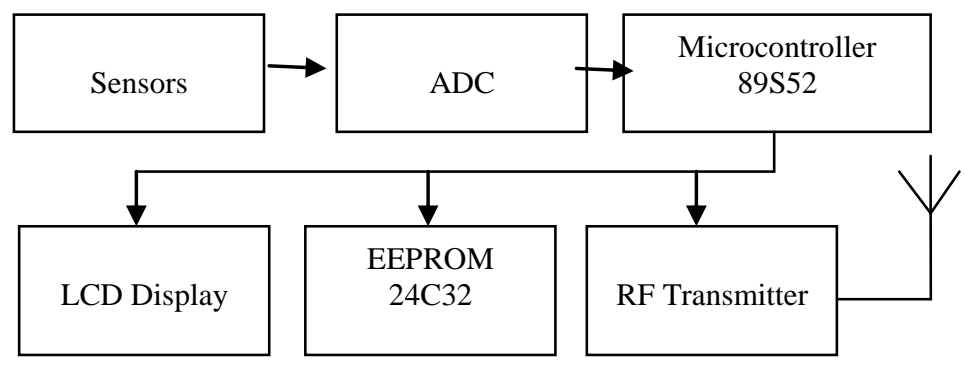

Fig 3 Transmitter circuit of coal mine system

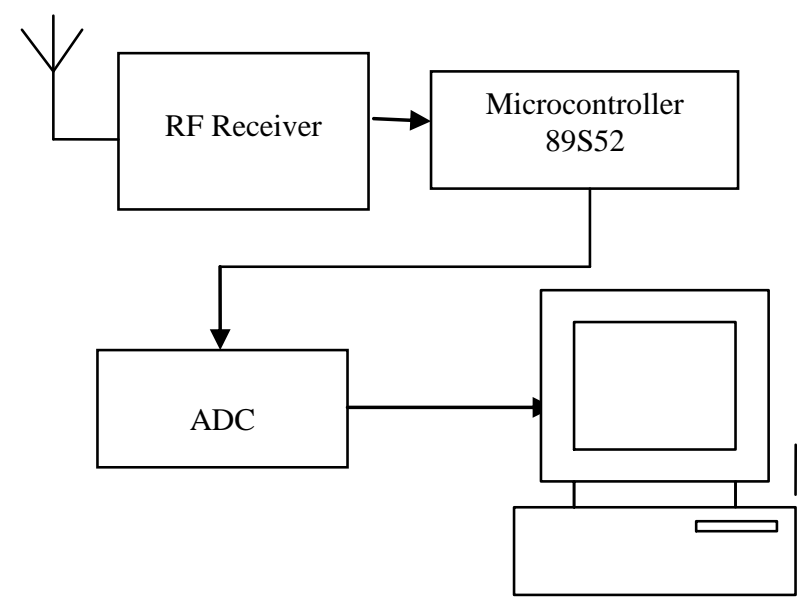

Fig. 4 Receiver Circuit Monitoring System

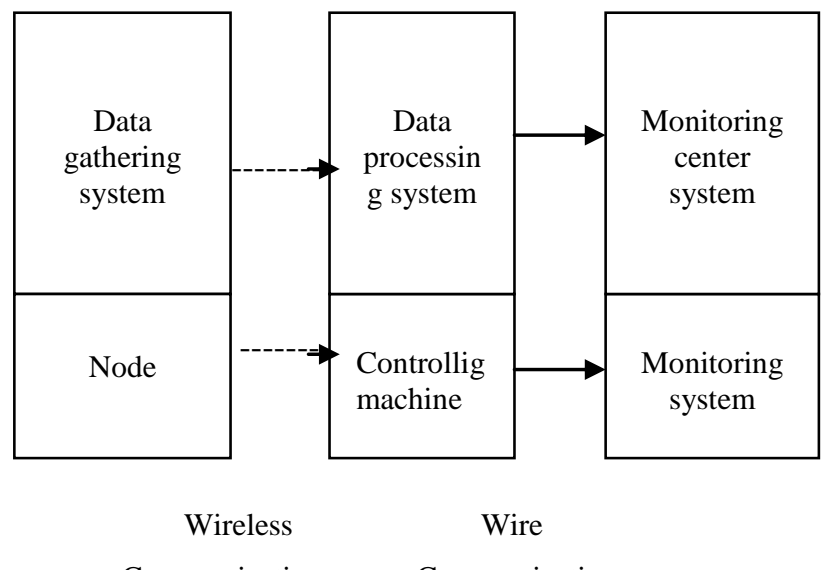

Communication Communication

Fig. 5 Monitoring System 
The system is consisted by three sub-systems: the data collecting sub-system, the data processing sub-system and the monitoring sub-system. The data collecting system contains the sensor nodes and the wireless communication parts, using for real-time gas concentration under ground monitoring. The collecting nodes are divided into two parts, movable nodes and static nodes. The movable nodes are responsible for the within its region and transmit the information collecting of information with other nodes; the statistic nodes are used for the summarizing of the region data and then transmit the summarized data to the main controlling system above ground. The data processing system contains the main controlling computer above ground and wire communication network using for the gathering, processing storing and transmitting of the information from all the lanes. The main controlling computer then transmits the final data to remote monitoring system. Remote monitoring system provides well man-machine interface. It stores the information received and show it in web way to the users.

\section{RESULT}

Coal Mine Security System totally based on wireless sensor network which consist of transmitter circuit and receiver circuit. In transmitter circuit sensor sense the given parameter and send this to analog to digital converter, then send this signal to 89S52 processor. Processor performs three functions.

- Transmit data

- Display data on monitor

- Store data permanently

Receiver receives data from transmitter, send it to 89S52 processor and finally send it on terminal as well as store it in a database system.

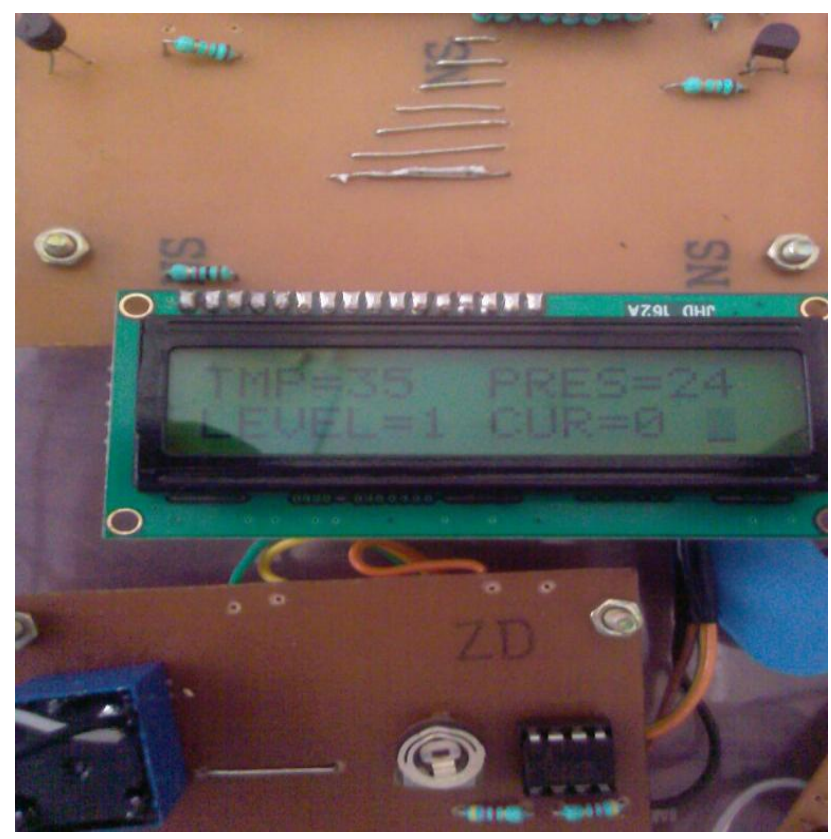

Fig.6 Monitoring Result

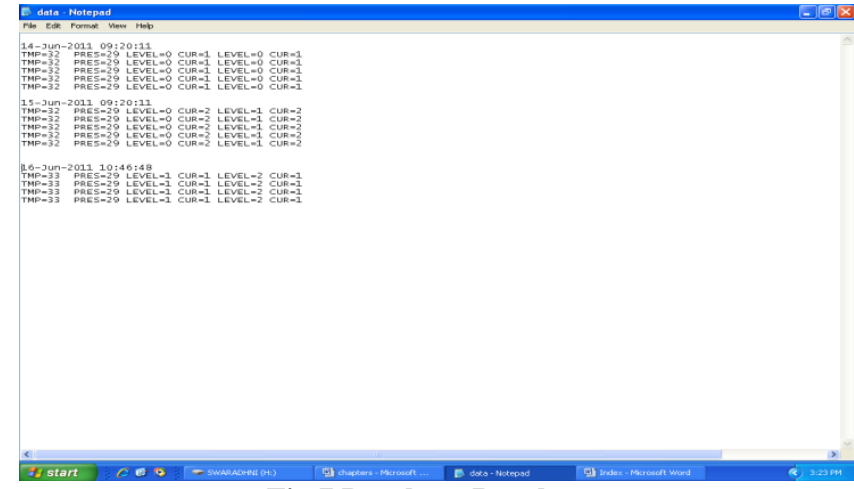

Fig.7 Database Result

\section{CONCLUSION}

Utilizing sensor technology, automatic detection Technology, communication technology and microcomputer technology, to realize the operational parameter intelligent monitored management of entire mining area, this system occupies following characteristic:

(1) The real-time data warning. The warning displays with kinds of representation. Using industry camera, it carries on image gathering and the remote control.

(2) Establish the real-time monitor security information data platform, Using the Matlab, making the system safety, convenient processing each kind data.

3) The expert decision - making support module sets in the system, can complete the Exide current, the mechanical device breakdown troubleshooting; Also may in the accident situation, provides the decision - making to rescue and disperse the personnel and equipment.

This Coal Mine Monitor System satisfies the user's request in the field of mine pit production condition real-time monitoring and dangerous situation discovery and elimination promptly, can cease the most mine accident occurrence effectively, provides a novel monitor method for the middle and small scale coal mine.

\section{REFERENCES}

[1] Warsha M choudhari,S.U.Nimbhorkar "An Intelligent Coal Mine Security System Using Multi-view Method",IJAEST Volume No.3 Issue No.1,15-19,2010

[2] LIU Tao ,HOU Yuan-bin,QI AI-ling,CHANG Xintan,"Study of Mine Information Security Based on multyview Method,"Xi an University of science and technology,China.2009.

[3] Song Dongdong,Ren Zhenhui,Gu Yanxia,’Design and Implement of An Intelligent Coal Mine Monitoring System,"2007.

[4] Zhanglin Guo,Chao Zhang,Jing Zhang,"The Study of Coal Mine Safety State Warning and Assistant Decisionmaking Support System's Construction,"2008.

[5] Pan Qi-dong,Zang Rui-xin,Duan Dong-sheng,Sun Gang,"Data Integration Research of Coal Mine Safety 
Production System for Emergency Decisionmaking,"2009.

[6] Yongiian Fan,Jianying Mdai,Yanguang Shen,"Study of the Safety Assesment Mode of Coal Mine Based on BP Neural Network,"Hebei University of Engineering,2009

[7] Wei Tan,Qianping Wang, Hai Huang ,Yongling Guo,Guoxia Zhang,"Mine Fire Detection Based on Wireless Sensor Network,"China University of Mining and Technology,July 9-11,2007.

[8] Chad O. Hargrave,Jonathon C. Ralston, and David W. Hainsworth,"Optimizing Wireless LAN for Longwall Coal Mine Automation ,"January/February 2007.

[9] Sun Yanjing, Qian Jiansheng, Li Shiyin and Song Jinling,"Intelligent Preduction System of Coal-Gas Outbrust Based on Evolutionary Neural Nets,'July 1621,2006 .
[10] Yifei Weng ,Lin Zhang,and X inzhe Jiu,"Supply Security simulation for the Coordination Mechanism of Coal Property-Right and Product Markets",2008.

[11] Zhu Yu ,Zhang Hong,Kong Ling-dong,”Research of Coal -Gas Outburst Forecasting Based on Artificial Immune Network Clustering Model", China University of mining and Technology,2009.

[12] Xiaosheng Liu,Ping Xue,"Pre-warning System of Mine Safety Based on Neural Network and expert System" jianjxi university of science and technology,2008.

[13] Zheng you, Guohuan Lou,j iawei Wang,Yuan Zhou,"Design of Coal Mechanical Online Fault Diagnosis based Embeded System",Hebei Polytechnic University,China,2010

[14] Yifei weng,lin Zhang,and Xinzhe Liu,"Supply Security System Simulation for the coordination of Coal propertyright and Product Markets"2008. 